\title{
Human 5-, 12- and 15-lipoxygenase-1 coexist in kidney but show opposite trends and their balance changes in cancer
}

\author{
AMIRA GOHARA $^{1}$, NOHA ELTAKI ${ }^{1}$, DINA SABRY ${ }^{1}$, DANIEL MURTAGH $\mathrm{Jr}^{2}{ }^{2}$, JERZY JANKUN $^{2-4}$, \\ STEVEN H. SELMAN ${ }^{2}$ and EWA SKRZYPCZAK-JANKUN ${ }^{2}$
}

\author{
Departments of ${ }^{1}$ Pathology and ${ }^{2}$ Urology, College of Medicine, University of Toledo, Toledo, OH 43614, USA; \\ ${ }^{3}$ Department of Clinical Nutrition, Medical University of Gdańsk, 80-211 Gdańsk, Poland; ${ }^{4}$ Protein Research Chair, \\ Department of Biochemistry, College of Sciences, King Saud University, Riyadh 11451, Saudi Arabia
}

Received May 4, 2012; Accepted June 21, 2012

DOI: $10.3892 /$ or.2012.1924

\begin{abstract}
Lipoxygenases make an impact on every stage of cancer affecting carcinogenesis, metastasis and apoptosis. While there is a rich literature on individual lipoxygenases we lack extensive data on their coexistence and balance in different organs and types of cancer. Renal cell carcinoma (RCC) is the most common type of kidney cancer in adults, characterized by a lack of early warning signs, diverse clinical manifestations, resistance to radiation and chemotherapy. One third of patients will relapse and the 5-year survival rate is $<10 \%$ in cases of metastases. Many drugs are metabolized in the kidneys and might interact with lipoxygenases that are biocatalysts for many endo- and xenobiotics. In the present study, we examined the kidney tissue from healthy individuals and cancer patients by immunohistochemical analysis for the presence of 3 lipoxygenases: 5-LOX, 12S-LOX and 15-LOX-1. Our findings confirmed their coexistence and opposite trends of manifestation in the course of disease with increased 15-LOX-1 and decreased 5and 12-LOX levels at the onset of cancer reversing with the progressing stage of the disease or the grade of tumor. Unlike other malignancies, there are no biomarkers to individualize RCC management. Modern therapies are using TKI therapy, targeting VEGF and may cause hypertension as a side-effect. 12S-LOX is intertwined with kinases and VEGF and increased secretion of 12S-HETE in urine is known to accompany hypertension. Thus, it may be valuable to probe $12 \mathrm{~S}$-LOX activity and monitor its natural metabolite to seek a possible aid in directing the treatment of patients.
\end{abstract}

\section{Introduction}

The kidney is one of the most essential organs in the human body. It maintains the homeostatic balance of fluids and

Correspondence to: Dr Ewa Skrzypczak-Jankun, Department of Urology, Urology Research Center, University of Toledo, Health Science Campus, 3000 Arlington Ave., Toledo, OH 43614, USA

E-mail: ewa.skrzypczak-jankun@utoledo.edu

Key words: human lipoxygenases, renal carcinoma, specific antibodies, RCC management, hypertension solutes, removes waste products from the blood, regulates blood pressure and secretes several hormones. The kidney is composed of a parenchyma and a collecting system, in which the parenchyma is composed primarily of nephrons, the functional filtering units of glomeruli and tubules, lined by glandular cells, and the collecting system includes the renal pelvis and calyces, lined by transitional cells.

Renal cell carcinomas ( $\mathrm{RCC}$ ) arise in the renal parenchyma, while those that arise from the collecting system are transitional cell carcinomas. In RCC, malignant cells originate in the lining of the proximal convoluted tubules, grow into mass within kidney or metastasize to other organs (1).

Kidney cancer is among the 10 most common cancers in both men and women. Overall, the lifetime risk for developing kidney cancer is about 1 in 67 (1.49\%). This risk is higher in men than in women. The American Cancer Society estimates that in 2011, there will be 60,920 new cases of kidney cancer (37,120 in males and 23,800 in females) with male to female ratio $\sim 3: 2$ and slightly higher odds in death cases for males (2). These statistics include both renal cell carcinomas and transitional cell carcinomas of the renal pelvis. The average age at diagnosis is 64 years. Kidney cancer is very uncommon in individuals younger than 45 , and it most often occurs in individuals of age 55 and older.

Renal cancer is characterized by a lack of early warning signs and diverse clinical manifestation. There are no recommended screening tests for kidney cancer. It is often found incidentally during CT or ultrasound scans performed for concerns other than renal cancer and thus detected when the primary tumor is already of considerable size (according to TNM system $\mathrm{T} 1$ is a tumor $\leq 7 \mathrm{~cm}$ across). The current treatment of choice is nephrectomy, i.e., removal of all or part of the kidney with the 5-year survival rate of $91 \%$ in low risk to $55 \%$ in high risk patients. Radiation and chemotherapy are not effective for treating RCC and although the progress has being made with contemporary targeted therapy in case of metastatic renal cell carcinoma (mRCC, 30-40\% of cases) the median overall survival ranges from several months for patients who show progression of cancer at 6 months, to $\sim 2$ years for those who do not (3). Therefore we are still on a lookout for the biomarkers and targets that might help to detect and treat renal carcinoma. 
Human lipoxygenases (LOXs) are widely distributed in a human body and have been localized in many organs, tissues and cells including kidneys (4-6). These enzymes catalyze peroxidation of unsaturated fatty acid producing variety of eicosanoids and are implicated in many diseases including cancer $(7,8)$. They also act on many endo- and xenobiotics $(9)$ including drugs passing through the kidneys. Several human lipoxygenases have been characterized (5-LOX, 12S-LOX, 15-LOX-1, 15-LOX-2 and epidermal 12R-LOX and e-LOX3). Experimental studies suggest that many cancers are driven by lipoxygenases and their metabolites HETEs (hydroxyeicosa-5,8,10,14-tetraenoic acid) but usually such experiments are directed to study only one particular LOX or relevant eicosanoid. We lack information on their coexistence: Is there anyone that dominates? What is their relation to one another and does the balance change in the course of the disease? How does it compare with the healthy tissue? The goal of this study was to detect three lipoxygenases 5-, 12S- and 15-LOX-1 in the same kidney tissue, from healthy people and cancer patients and attempt to find the answers to the above questions.

\section{Materials and methods}

Human tissue samples. Blocks of paraffin-embedded kidney tissue were selected from the archives of the Department of Pathology, representing normal kidney from healthy individuals (NK, 13), normal looking tissue from cancerous organ (NKC, 17), cases diagnosed as renal clear cell carcinoma (RCC, 31), renal papillary carcinoma (RPC, 23) and for a total of 60 patients but 84 specimens with 49 male and 35 female. 'Other' renal cancers such as sarcoma, chromophobe and unclassified were rejected.

Immunohistochemistry. Consecutive 4- $\mu \mathrm{m}$ slides were cut from paraffin blocks, mounted and manually stained with primary antibodies to detect 5-LOX, 12-LOX and 15-LOX-1, and no primary antibody (PBS instead) for the negative controls, using R.T.U. Vectastatin Universal kit, DAB kit, hematoxylin and VectaMount non-aqueous mounting media (Vector Laboratories, Inc., Burlingame, CA). Warming time (15-35 min), concentration (up to $10 \mu \mathrm{g} / \mathrm{ml}$ ) and the incubation time (1-3 h) for each primary antibody were tested and samples stained following the established protocol. Specimens were deparaffinized and rehydrated by heating at $60^{\circ} \mathrm{C}$ in an oven for $30 \mathrm{~min}$, washed in xylene twice for $5 \mathrm{~min}$, followed by washes in $\mathrm{EtOH} 100 \%$ $\rightarrow 95 \% \rightarrow 90 \% \rightarrow 80 \% \rightarrow 70 \% \rightarrow 50 \% \rightarrow 25 \%$ for $2 \mathrm{~min}$. They were covered with blocking solution (normal horse serum 2.5\%), incubated for $10 \mathrm{~min}$ in a humid box, followed by incubation with the primary antibody $(10 \mu \mathrm{g} / \mathrm{ml}$ with $1.5 \% \mathrm{v} / \mathrm{v}$ of blocking solution or PBS in case of negative control) at $37^{\circ} \mathrm{C}$ in $91 \%$ humidity chamber, 10 min with secondary antibody (biotinylated pan-specific antibody, anti-mouse/rabbit/goat IgG $(\mathrm{H}+\mathrm{L})$ made in horse) and $10 \mathrm{~min}$ with strepavidin/peroxidase complex, in a humid box at the room temperature. Each step was alternated by 2 washes in PBS for 2 min each. All slides were stained with DAB (1:2:1 v/v of the buffer stock solution, DAB stock solution and $\mathrm{H}_{2} \mathrm{O}_{2}$ ) for 5 min in humid box, washed in PBS for $2 \mathrm{~min}$, water for $30 \mathrm{sec}$, then counterstained with hematoxylin for $1 \mathrm{~min}$, washed in running water, PBS washed for $45 \mathrm{sec}$ and rehydrated by immersing for $2 \mathrm{~min}$ in $90 \%$
Table I. Age of patients in the evaluated groups.

\begin{tabular}{lcr}
\hline Group & Average age & Range \\
\hline Normal $^{\mathrm{a}}$ & $48 \pm 23$ & $7-88$ \\
NKC & $61 \pm 11$ & $39-75$ \\
Cancerous & $68 \pm 16$ & $31-86$
\end{tabular}

Normal, healthy; NKC, normal tissue from a cancerous organ. ${ }^{\text {aThree }}$ individuals were 7,12 and 18, thus the high spread and high standard deviation ( $59 \pm 13$ without these three).

$\rightarrow 95 \% \rightarrow 100 \% \mathrm{EtOH}$ and 2 washes of xylene, 3 min each. They were preserved with mounting medium and air dried at room temperature.

Antibodies. The primary antibodies against 5-LOX, 12S-LOX, 15-LOX-1 were selected from those commercially available using information about their specificity and the lack of cross-reactivity as a guidance. Polyclonal rabbit antibody ab39347 developed against a 130-149 sequential fragment of 5-LOX (does not react with human 12- or 15-LOX, tested for IHC-P application) and monoclonal murine antibody ab54772 based on whole length 15-LOX-1 (claimed as specific but not tested for cross-reactivity or IHC-P application), were from Abcam, Cambridge, MA. Murine monoclonal antibody 7225 developed against whole length recombinant 12S-LOX was from American Diagnostica, GmbH, Pfungstadt, Germany, tested against all recombinant human lipoxygenases, i.e., 5-, 12S-, 12R-, 15-type 1 and 15-type 2 and e-LOX3, proven 12S-LOX specific and successfully tested for IHC-P application on various normal and cancerous tissues (Dr J. Reinhardt, American Diagnostica). Except 12S-LOX (produced in our laboratory) other LOXs were generous gifts from Dr T. Holman, University of California, Santa Cruz, CA (5-, 15-LOX-1 and -2) and Dr A. Brash, Vanderbuilt University, Nashville, TN (12R-LOX and e-LOX3).

Other chemicals. Xylene histological grade was purchased from Sigma-Aldrich, PBS 10X from Fisher Scientific, ethyl alcohol 100\% from Pharmco-AAPER.

Specimen evaluation. The extent and intensity of staining was graded on scale $0-4$ by two observers in a blinded manner using coded slides. After the slides had been evaluated the code was broken, the cases grouped according to the tissue type, cancer description and patient gender, and the data compared for 3 antibodies and corresponding negative controls. A pathologist evaluated staining in epithelial and stromal cells, blood vessel endothelium, focal vs. diffuse, kidney tubules vs. glomeruli and membranous vs. cytoplasmic or nuclear location. All normal or cancerous tissue on the slide was taken into account to assign the final score.

The data were tabulated, grouped according to disease, cancer TNM stage and tumor grade (10). Only specimens of normal kidney (NK), normal looking tissue from cancerous kidney (NKC) and those with renal clear cell carcinoma (CC1 and $\mathrm{CC} 3$ ) and renal papillary carcinoma (PC1 and $\mathrm{PC} 3$ ) were 


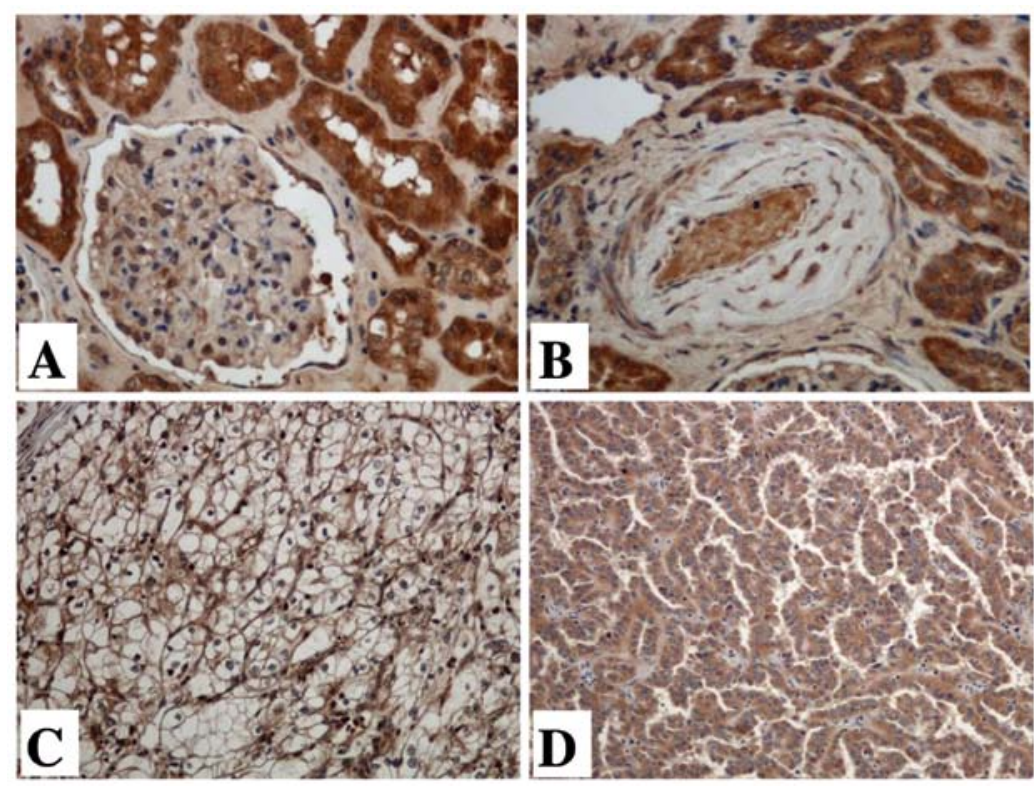

Figure 1. Positive staining with 7225 (against 12-LOX). Normal kidney tissue at $\mathrm{x} 40$ magnification. (A) Kidney tubules and glomeruli, (B) of a blood vessel in normal kidney tissue at x100 magnification, (C) clear cell carcinoma at x40 magnification and (D) papillary carcinoma at x40 magnification.
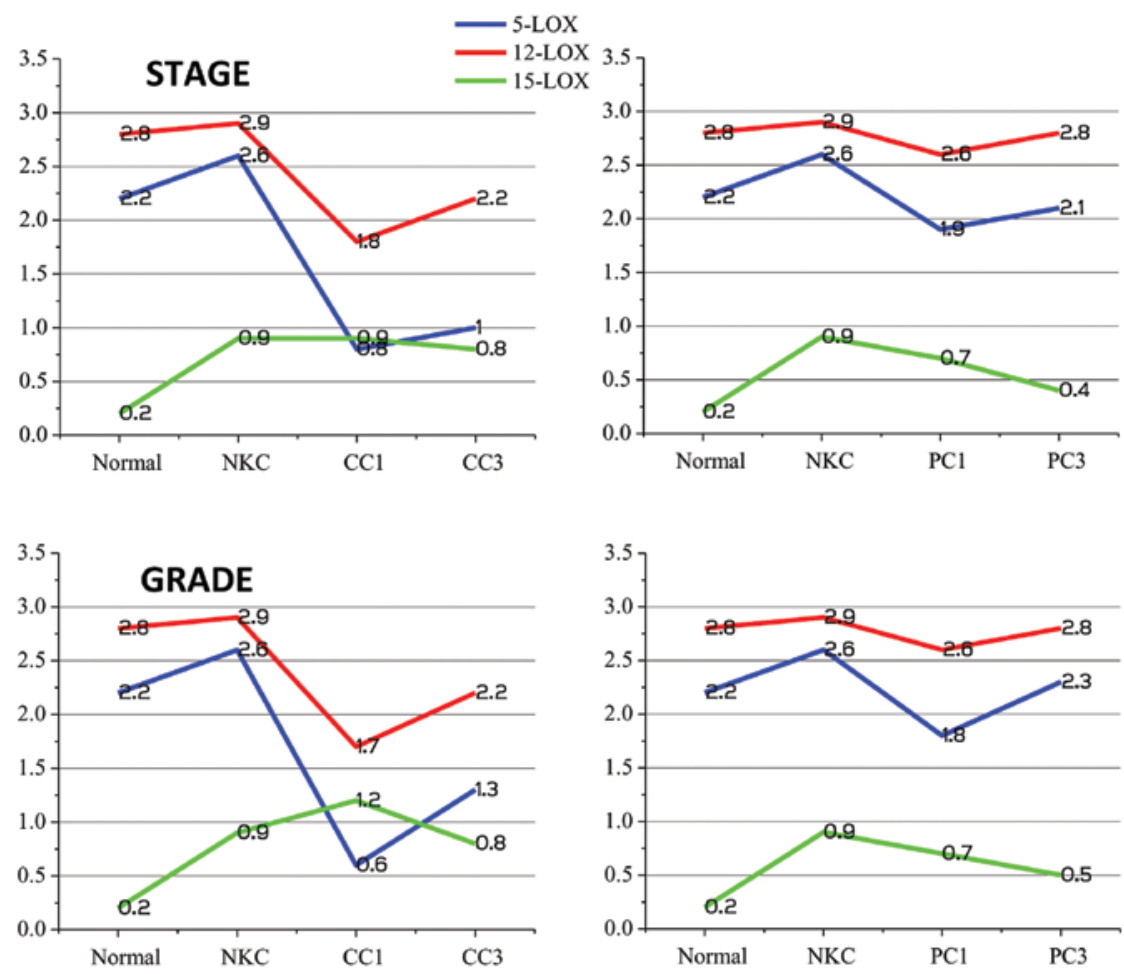

Figure 2. Trends in intensity of staining for 5-, 12- and 15-LOX in healthy tissue (NK), normal tissue in cancerous kidney (NKC) vs. clear cell carcinoma (left) and papillary carcinoma (right) according to the stage and grade of tumor.

included in the statistics. In terms of 'stage' we considered pT1 and pT3 for early and advanced stage, $\mathrm{N}$ and $\mathrm{M}$ were mostly not assessed with only two samples given N0 and two N1. In terms of grade the samples were grouped as 1-2 as one group, and 3-4 as another with majority of 1 and 3. Statistical analysis was performed as one way analysis of variance (ANOVA) followed by Duncan's multiple comparison test by the SAS v.9.1 program (SAS Institute, Cary, NC).

\section{Results}

Results are illustrated in Fig. 1 and summarized in Tables I, II and III and Fig. 2. From all the specimens viewed under the light microscope, it was evident that 5-, 12S- and 15-LOX-1 were all present in endothelial cells, cytoplasm and membranes of the proximal and distant tubules. Sporadic and focal staining was observed in glomeruli only in NK, negative staining was 
Table II. Intensity of staining for 5-LOX, 12S-LOX and 15-LOX-1 according to stage or grade of tumor.

\begin{tabular}{lcccc}
\hline Tissue & No. & 5 -LOX & 12 -LOX & 15 -LOX \\
\hline Normal kidney & 13 & $2.2 \pm 1.3$ & $2.8 \pm 1.1$ & $0.2 \pm 0.3$ \\
Normal looking cancer tissue & 17 & $2.6 \pm 1.0$ & $2.9 \pm 1.1$ & $0.9 \pm 1.4$ \\
Clear cell carcinoma & & & & \\
$\quad$ Stage & & & & $0.9 \pm 1.5$ \\
pT1 & 14 & $0.8 \pm 1.1$ & $1.8 \pm 1.3$ & $0.8 \pm 1.3$ \\
pT3 & 17 & $1.0 \pm 1.1$ & $2.2 \pm 1.2$ & $1.2 \pm 1.6$ \\
Grade & & & & $0.3 \pm 0.8$ \\
1-2 & 15 & $0.6 \pm 0.8$ & $1.7 \pm 1.4$ & \\
3-4 & 16 & $1.3 \pm 1.2$ & $2.2 \pm 1.1$ & $0.7 \pm 1.3$ \\
Papillary carcinoma & & & & $0.4 \pm 0.9$ \\
Stage & 16 & $1.9 \pm 1.2$ & $2.6 \pm 1.1$ & $0.7 \pm 1.3$ \\
pT1 & 7 & $2.1 \pm 1.1$ & $2.8 \pm 0.9$ & $0.5 \pm 1.2$ \\
pT3 & & $1.8 \pm 1.1$ & $2.6 \pm 1.1$ & $2.8 \pm 1.0$ \\
Grade & 14 & $2.3 \pm 1.0$ & & \\
1-2 & 9 & & & \\
3-4 & & & & \\
\hline
\end{tabular}

Table III. Comparison of data for gender.

\begin{tabular}{lccc}
\hline Tissue & $\mathrm{M} / \mathrm{F}$ & $\mathrm{M}$ & $\mathrm{F}$ \\
\hline Normal & & & \\
$\quad$ Healthy & $16 / 10$ & $2.4 \pm 1.4$ & $2.6 \pm 1.0$ \\
$\quad$ Cancer patient & $22 / 12$ & $2.6 \pm 1.0$ & $3.0 \pm 1.1$ \\
Clear cell carcinoma & & & \\
$\quad$ Early & $12 / 18$ & $1.2 \pm 1.2$ & $1.3 \pm 1.3$ \\
$\quad$ Advanced & $18 / 14$ & $1.5 \pm 1.2$ & $1.8 \pm 1.3$ \\
Papillary carcinoma & & & \\
$\quad$ Early & $20 / 8$ & $2.0 \pm 1.2$ & $2.5 \pm 1.1$ \\
$\quad$ Advanced & $8 / 10$ & $2.5 \pm 1.0$ & $2.4 \pm 1.1$ \\
\hline
\end{tabular}

M, male; F, female. Lipoxygenases 5- and 12- with the same trend are grouped together.

present in stroma and blood vessels and positive staining was present in smooth muscle in some cases. The differences were noted according to the Tukey's studentized range test for intensity are shown in Table IV.

Presence of lipoxygenase. From this study it is evident that lipoxygenases coexist in the kidney tissue, and there are no distinctive differences between the three LOXs regarding localization in renal tissue, that is no preference of staining in stroma, cells or nucleus. It is also shown that 12-LOX consistently dominates over 5-LOX and 15-LOX-1 in normal and cancerous renal tissues: $12-\mathrm{LOX}>5-\mathrm{LOX}>>15-\mathrm{LOX}-1$, except for renal clear cell carcinoma where 5- and 15-LOX do not significantly differ in terms of 'stage' category. Furthermore, it is shown that only in the very advanced stage of cancer may the level of lipoxygenase approach or exceed its presence in normal tissue (Table II and Fig. 2).
Table IV. Results of the Turkey's studentized range test for intensity of 5-LOX, 12-LOX and 15-LOX in the various samples.

\begin{tabular}{lcccccc}
\hline & $\mathrm{NK}$ & $\mathrm{NKC}$ & $\mathrm{CC} 1$ & $\mathrm{CC} 3$ & $\mathrm{PC} 1$ & $\mathrm{PC} 3$ \\
\hline 5-LOX & $\mathrm{A}$ & $\mathrm{A}$ & $\mathrm{A} / A$ & $\mathrm{~A} / A$ & $\mathrm{~A} / A$ & $\mathrm{~A} / A$ \\
12-LOX & $\mathrm{A}$ & $\mathrm{A}$ & $\mathrm{B} / B$ & $\mathrm{~B} / A$ & $\mathrm{~A} / A$ & $\mathrm{~A} / A$ \\
15-LOX & $\mathrm{B}$ & $\mathrm{B}$ & $\mathrm{A} / B$ & $\mathrm{~A} / B$ & $\mathrm{~B} / B$ & $\mathrm{~B} / B$ \\
\hline
\end{tabular}

Significant differences at $\mathrm{P}<0.05$ were observed when marked by a different letter [stage of disease/grade of tumor (italics)].

Differences between lipoxygenases. 12-LOX and 5-LOX behave similarly. The levels of 5-LOX and 12-LOX decline at the onset of cancer, but show increased expression with higher grade tumors or progression of the disease. On the other hand, 15-LOX-1 indicates an opposite trend to 5- and 12S-LOX, showing higher level at the onset of cancer and lower with higher grade of tumors or progression of disease (in case of papillary carcinoma). Contrary staining of 15-LOX-1 appears to be absent, or very weak in NK and grade 3 in CC3 or PC3, but could be stronger in grade 1 of $\mathrm{CC} 1$ and grade 4 of 'other' cancers (that were stained and evaluated but not included in the statistics).

Gender. We did not observe significant differences related to gender (Table III).

\section{Discussion}

Renal cell carcinoma is the most common type of kidney cancer, accounting for $\sim 90 \%$ of all malignant kidney tumors. Out of the $90 \%$, there are five main types of renal cell carci- 
Table V. Human Protein Atlas (6) - normal kidney and cancer, data for ALOX5, ALOX12 and ALOX15 for the antibody of the highest reliability.

\begin{tabular}{|c|c|c|c|c|c|c|c|c|c|c|c|c|c|c|}
\hline \multirow[b]{2}{*}{ Patient ID } & \multicolumn{2}{|c|}{$\begin{array}{c}\text { Patient } \\
\text { characteristics }\end{array}$} & \multicolumn{4}{|c|}{$\mathrm{ALOX5}^{\mathrm{a}}$} & \multicolumn{4}{|c|}{ ALOX $12^{\mathrm{b}}$} & \multicolumn{4}{|c|}{$\operatorname{ALOX} 15^{\mathrm{a}}$} \\
\hline & Cender & Age & S & I & $\mathrm{Q} \%$ & Loc & $\mathrm{S}$ & I & Q \% & Loc & $\mathrm{S}$ & I & Q \% & Loc \\
\hline \multicolumn{15}{|c|}{ Normal kidney } \\
\hline 1933 & F & 56 & M & M & $75-25$ & $\mathrm{CM}$ & M & M & $>75$ & $\mathrm{CM}$ & M & M & $>75$ & $\mathrm{CM}$ \\
\hline 2165 & M & 7 & $\mathrm{M}$ & $\mathrm{M}$ & $75-25$ & $\mathrm{CM}$ & $\mathrm{M}$ & $\mathrm{M}$ & $>75$ & $\mathrm{CM}$ & ND & & & \\
\hline 1943 & M & 28 & M & M & $75-25$ & $\mathrm{CM}$ & ND & & & & ND & & & \\
\hline 1767 & M & 16 & ND & & & & ND & & & & M & $\mathrm{M}$ & $>75$ & $\mathrm{CM}$ \\
\hline 3229 & M & 59 & ND & & & & $\mathrm{M}$ & M & $>75$ & $\mathrm{CM}$ & M & M & $>75$ & $\mathrm{CM}$ \\
\hline \multicolumn{15}{|c|}{ Kidney cancer } \\
\hline 1722 & $\mathrm{~F}$ & 60 & M & M & $75-25$ & $\mathrm{CM}$ & M & M & $>75$ & $\mathrm{CMN}$ & - & - & - & - \\
\hline 1498 & $\mathrm{~F}$ & 70 & & & & & $\mathrm{~W}$ & W & $>75$ & $\mathrm{CM}$ & - & - & - & - \\
\hline 2067 & F & 72 & M & M & $75-25$ & $\mathrm{CM}$ & ND & & & & M & M & 75 & $\mathrm{CM}$ \\
\hline 2564 & $\mathrm{~F}$ & 52 & M & M & $>75$ & $\mathrm{CM}$ & $\mathrm{M}$ & $\mathrm{M}$ & $>75$ & $\mathrm{CM}$ & M & $\mathrm{M}$ & $>75$ & $\mathrm{CM}$ \\
\hline 3061 & F & 57 & W & M & $<25$ & $\mathrm{CM}$ & M & M & $>75$ & $\mathrm{CMN}$ & - & - & - & - \\
\hline 1901 & $\mathrm{~F}$ & 69 & - & $\mathrm{W}$ & $<25$ & $\mathrm{CM}$ & $\mathrm{M}$ & $\mathrm{M}$ & $>75$ & $\mathrm{CM}$ & ND & & & \\
\hline 1352 & $\mathrm{~F}$ & 83 & - & - & - & - & ND & & & & ND & & & \\
\hline 59 & M & 2176 & $\mathrm{~W}$ & W & $75-25$ & $\mathrm{CM}$ & $\mathrm{M}$ & M & $>75$ & $\mathrm{CM}$ & $\mathrm{M}$ & M & 75 & $\mathrm{CM}$ \\
\hline 56 & M & 1752 & M & M & $>75$ & $\mathrm{CM}$ & $\mathrm{W}$ & $\mathrm{W}$ & $>75$ & $\mathrm{CM}$ & - & $\mathrm{W}$ & $<25$ & $\mathrm{CM}$ \\
\hline 68 & M & 2452 & $\mathrm{~W}$ & M & $<25$ & $\mathrm{CM}$ & M & M & $75-25$ & $\mathrm{CM}$ & $\mathrm{W}$ & M & $<25$ & $\mathrm{CM}$ \\
\hline 77 & M & 1831 & M & M & $>75$ & $\mathrm{CM}$ & $\mathrm{W}$ & W & $>75$ & $\mathrm{CM}$ & M & M & 75 & $\mathrm{CM}$ \\
\hline 63 & M & 1969 & M & M & $>75$ & $\mathrm{CM}$ & W & W & $>75$ & $\mathrm{CM}$ & M & M & $>75$ & $\mathrm{CM}$ \\
\hline 61 & M & 2540 & $\mathrm{~S}$ & $\mathrm{~S}$ & $75-25$ & $\mathrm{CMN}$ & $\mathrm{M}$ & $\mathrm{M}$ & $>75$ & $\mathrm{CM}$ & M & $\mathrm{M}$ & $>75$ & $\mathrm{CM}$ \\
\hline
\end{tabular}

S, antibody staining. I, intensity given as: -, negative or none; W, weak; M, medium; S, strong. Q, quantity, means \% of cells showing staining. Loc, location: C, cytoplasmic; M, membranous; N, nuclear; ND, no data. Antibodies: for ALOX5, CAB005066 (Santa Cruz Biotechnology SC-8885) supportive for IHC; for ALOX12, HPA010691 (Sigma-Aldrich) supportive for IHC; for ALOX15, HPA013859 (Sigma-Aldrich) IHC uncertain. ${ }^{a}$ Negative in glomeruli, positive in tubules of normal kidney. ${ }^{b}$ Localized in glomeruli and tubules of normal kidney.

noma that are identified by microscopic examination: clear cell, papillary, chromophobe, oncocytoma and 'unclassified'.

Clear cell (CC) carcinoma is the most common form of renal cell carcinoma, accounting for about $80 \%$ of cases with kidney cancer. When viewed under a microscope, the individual cells that make up clear cell carcinoma appear clear and very pale. Clear cell carcinoma is a renal cortical tumor characterized by malignant epithelial cells with clear cytoplasm and a 'compact-alveolar or acinar growth pattern'. A variable proportion of cells with granular eosinophilic cytoplasm may be present. Unlike renal clear cell carcinoma, papillary carcinoma (PC) is the second most common type accounting for about $10-15 \%$ of cases. These cancers form little fingerlike projections, called papillae (Fig. 1). Papillary renal cell carcinomas are a less aggressive type of kidney cancer, usually well encapsulated, grow slowly and have an overall lower malignant potential. Classifications for the non-clear cell renal carcinomas (papillary carcinoma and others) are continuously adjusted according to new cytogenetic and molecular data and their diagnosis depends on studying various morphological and immunohistochemical criteria (11). Discussion of our results is restricted to renal cancers of CC and PC category.
Lipoxygenases are known to be involved in carcinogenesis but studies are usually restricted to one type of enzyme and we lack information on their coexistence and mutual relation. 5- and 12-LOX reveal pro-carcinogenic properties, while 15-LOX-1 and especially induced 15-LOX-2 show anticarcinogenic roles in published research studies (12). For renal carcinoma we have found two examples of immunohistochemical evaluation for 5-LOX $(13,14)$, one for 12-LOX $(15)$ and none for 15-LOX except examples in the Human Protein Atlas (6). Below is a summary of the available data on renal carcinoma and LOX.

5-Lipoxygenase. Faronato et al examined 46 snap-frozen primary renal cell carcinomas and their corresponding normal cortex biopsies. It was described that quantitative 5-LOX mRNA analysis by RT-PCR showed up to 3-fold increased level of expression in the tumor tissue. However, the relative amount of 5-LOX mRNA in 32 samples was 9.53-12.48 with median 11.13 in tumor tissue and 11.25-14.65 with median 13.15 in paired normal tissue, thus this conclusion is confusing. The results from immunoblotting (WB) positively correlate with the tumor grade $(\mathrm{P}<0.01)$, pathological stage 
$(\mathrm{P}<0.05)$ and the patients age (in two groups below and above 60 years, $\mathrm{P}<0.002$ ). It has to be noted that the authors compared cancerous tissue with paired normal tissue and the score was calculated only for cases with visual difference and increased level of 5-LOX in the cancerous section. The 58 samples: 50 of clear cell carcinoma, 6 of papillary carcinoma and 2 of chromophobe were treated as one group (13). The antibody was from BD Biosciences, Oxford, UK, which was developed against human 5-LOX fragment 442-500 (out of 674 residues), monoclonal, purified by affinity chromatography, routinely tested and recommended for WB but the information sheet does not indicate testing for the cross-reactivity with other human LOXs (13).

12-Lipoxygenase. Yoshimura et al have done immunohistochemical analysis of paraffin-embedded samples: 108 of renal cell carcinoma and 20 from patients with normal kidney tissue. It is not clear if the cancerous tissue were all of 'clear cell' type or of varied characteristics and what stage or grade. The primary antibody against human 12-LOX was proprietary (no description given, albeit the authors mention that immunostaining with anti-12-LOX antibody absorbed with synthetic 12-LOX peptide was negative). In the normal kidney (NK) the authors report presence of 12-LOX in the collecting ducts, blood vessels and stroma but not in glomeruli. In tissue from renal cell carcinoma ( $\mathrm{RCC}$ ) the staining was strongly localized in the perinuclear and cytoplasmic regions but only weakly in nucleus. The evaluation done brought the average score on $0-4$ scale as $\sim 0.6$ for NK and $\sim 3.2$ for RCC in all three evaluated categories, i.e., epithelium, blood vessels and stromal tissue (15).

15-Lipoxygenase. We could not find any previous data for 15-LOX evaluation in renal carcinoma. However, there are studies on 15-LOX-1 in bladder cancer (16) and 15-LOX-2 in prostate cancer (17) where the authors claim using specific antibodies for this enzyme and in (16) the antibody was tested against human 5-LOX, 12-LOX, 15-LOX-2 and cyclooxygenases. In IHC analysis of paraffin-embedded bladder cancer samples a progressive decrease in protein level was observed with the increasing stage of the tumorigenic tissue, $0.86 \pm 0.09$ $(\mathrm{T} 3 / \mathrm{T} 4, \mathrm{n}=6)$ vs. $1.93 \pm 0.06$ in normal tissue $\mathrm{n}=24(\mathrm{P}<0.001)$. Also WB and RT-PCR showed higher enzyme expression in normal tissue vs. high stage tumor. In prostate cancer IHC study $(\mathrm{n}=70)$ bening glands showed uniform 15-LOX-2 immunostaining whereas in adenocarcinomas it was markedly reduced and it correlated with the tumor grade (17).

More than one enzyme. It is very rare to find data on more than one oxygenase in the characterized samples. Matsuyama et al examined kidney tissue in paraffin embedded samples for presence of 5- and 12-LOX. It was found that NK 5- and 12-LOX are observed only in tubules but not in epithelial cells, blood vessels or stroma. But in cancer specimens staining was strong for both enzymes, localized in the perinuclear and cytoplasmic regions, weak in the nucleus, and significantly declining with the increasing grade of tumor. In general staining in RCC tissue was $\sim 5$ times stronger than in NK. This analysis was done for 50 patients with renal cell carcinoma (clear cell, papillary, chromophobe and collecting duct carci- noma were treated as one group) and 10 patients with normal kidney tissue (however they all underwent total nephroureterectomy due to ureteral cancer). The primary antibodies were from Cayman Chemicals, USA against fragment 130-149 in human 5-LOX sequence, and from Oxford Biomedical Research, USA for 12-LOX (description not available). In the same publication nested RT-PCR showed significant expression of 5- and 12-LOX in cancer cells but none in NK cell (14). Jiang et al determined levels of expression of 5-, 12- and 15-LOX-1 as well as COX-2 in frozen tissues of human breast cancer by RT-PCR. In general the analyses showed high content of 5-LOX in normal tissue and somewhat lower level in cancerous, high of 12-LOX in cancer vs. none in normal, COX-2 even higher than 5-, 12-LOX in cancerous tissue with low in normal, while for 15-LOX-1 it was very high in normal and much lower in cancer. The authors made interesting observations about fluctuations of the enzyme levels depending on TNM and grade of tumor, local recurrence and metastasis. Tissue of patients who died of breast cancer expressed (results from RT-PCR given as: disease-free/cancer) very high level of 5-LOX $(\sim 60 / \sim 320)$, lower of 12-LOX (50/ 230), none of 15-LOX (200/0) and elevated COX-2 ( 40/ 100) (18). Yoshimura et al examined paraffin-embedded tumor specimens of bladder cancer $(n=170)$ by immunostaining for 5 - and 12-LOX. Normal samples of bladder tissue were used from patients $(n=20)$ who underwent prostatectomy due to prostate cancer. In general, intensity of staining was high in epithelium, blood vessels and stroma, somewhat higher for 5-LOX than 12-LOX and $>3$ times higher in cancer than normal tissue in all three categories. However, the statistical analysis (ANOVA) showed significant differences only for epithelium indicating progression with increasing grade ( 2 and higher) or stage (pT2 or greater) (19).

Human Protein Atlas deserves special attention (6). This database contains information on $>5,000$ human proteins, $>6,100$ antibodies and $>5$ mln images of immunohistochemical staining of human tissue from various organs. It contains information on source and validation of all antibodies and provides expression profiles in normal and cancer tissue and cell lines. It also proves what a conflicting results can be observed in case of non-specific and not fully tested antibody as can be seen for 15-LOX-1 (http://www.proteinatlas.org/ ENSG00000161905/normal/kidney). The data extracted from this database on 5-, 12- and 15-LOX-1 in normal kidney and cancer are given in Table V. It is still sparse but shows similar trend to our results.

In light of the observations made by others our results are consistent with the findings in the Human Protein Atlas database (Table V) in terms of 12-LOX dominating the picture followed by 5-LOX that occasionally can show stronger staining but in terms of score their differences are not significant $(\mathrm{P}<0.05)$. However, both 5 - and 12-LOX stain much stronger than 15-LOX-1. Our observations for 15-LOX agree with the trend observed in (16-18), i.e., decreasing level of enzyme with progress of cancer, however the cited results from literature were for bladder, prostate and breast cancer, not kidney, and also reported significantly higher level in NK than in carcinoma. It was mentioned in IHC for prostate cancer and 15-LOX-2 that negative immunostaining was observed in $>50 \%$ of the tumor cells (17). In our IHC analysis for 15-LOX-1 
we observed negative staining in a large percentage of cells, and in general weak staining when it was present. However, we have observed lower values for NK and $\sim 4 \mathrm{x}$ higher on the onset of cancer with decreasing tendency with progression of the disease, but statistically the differences were insignificant $(\mathrm{P}>0.05)$ except for CC1. In case of 5-LOX (13) and 12-LOX (15) low score $(<1)$ was reported for NK tissue and higher $(\sim 3)$ in cancerous samples (13-15). It is worth noting that evenly strong staining was observed in epithelium, blood vessels and stromal tissue both in NK ( 0.6) as well as RCC ( 2-3) $(14,15)$, which does not agree with our evaluation and the Human Protein Atlas data. However, only the epithelium score differ with the changing grade of tumor showing significant decrease $(\mathrm{P}<0.001)$ between grade $1(\sim 3)$ and grade $3(\sim 2)(14)$.

It is difficult to compare results when not using the same antibodies - the trend however should be the same. This cannot be said about 5-LOX and 12-LOX where the level of enzyme in cancer tissue was reported higher than in normal. While we agree that for 5- and 12-LOX it is increasing with the progression of the disease our average scores have the highest values for $\mathrm{NK} / \mathrm{NKC}$, then show decline in value at the onset of the disease in the cancerous tissue and climb later toward that of $\mathrm{NK}$ level, both in clear cell (CC) as well as papillary (PC) carcinoma. 15-LOX-1 shows a reverse trend. The difference between NKC and NK is not statistically significant but we have noted that NKC values do tend to be higher than NK from healthy individuals. Hence maybe the body is trying to counterbalance changes in the cancerous tissue by producing more in the healthy looking counterpart of the same organ. In such case the paired samples could have the elevated values in comparison with those found in cancer-free, healthy individuals. In a previous study (14) normal looking tissue of the kidneys removed from patients with transitional renal cancer were accepted as NK. Therefore, what was categorized as 'healthy' could be affected by cancer. In case of 5-LOX (13) the authors anticipated increased level in cancer and selected only these data. In case of 12-LOX (15) the staining was even 'across the board', i.e., in epithelium, blood vessels and stroma, both in NK and renal carcinoma, but varied only in epithelium when the tumor grade was taken into consideration (14).

Considering conflicting observations made in the studies described here one might put in question the specificity of the antibodies. In general the antibody could be developed against some arbitrary selected polypeptide instead of a whole length protein, it could be polyclonal or monoclonal, purification could be different and specificity not confirmed against other proteins that might have similar epitopes. The antibodies produced earlier were usually developed against short polypeptides anticipated to have an epitope distinguished for the given enzyme. They were seldom tested for cross-reactivity with other human lipoxygenases and were not commercially available (if proprietary) or are no longer available. Nowadays, reliable antibodies are usually developed against whole length enzyme, carefully purified, monoclonal and thoroughly tested for specificity. Our choice of the antibodies was dictated by the availability of this information. It might be worth mentioning that in testing antibodies developed against whole length recombinant platelet $12-\mathrm{LOX}$ we have noticed stronger staining for polyclonal antibodies than for monoclonal. Therefore, when comparing data for 5-LOX (polyclonal antibody) vs.
12-LOX (monoclonal) and 15-LOX-1 (monoclonal) we might have somewhat skewed our results in favor of 5-LOX.

The results from IHC analysis of paraffin embedded specimens, even if double blinded, may always be limited by the crude scale used in the evaluation. More accurate and objective data could be provided by RT-PRC by utilization of the fresh or snap frozen samples. From the literature available it is obvious that the level of a given LOX may be conditional on the organ/tissue and might strongly fluctuate depending on tumor characteristic and stage of the disease. In our study we have observed small changes in 5- and 12-LOX in papillary cell carcinoma (PC) and statistically insignificant, while in clear cell carcinoma (CC) they are more pronounced (Fig. 2). Cases of chromophobe showed the most intensive staining (and were not included in our results presented here). Thus in the studies where all types of tumors were taken together for the calculated average score in the given group (for example 'grade 1') might be affected by the content of this group if the number of different cancer types (clear cell, papillary cell, chromophobe and sarcoma) were not balanced.

Jiang et al (18) who studied 5-, 12-, 15-LOX and COX-2 in breast cancer concluded that breast tumors aberrantly express LOXs and COX-2 and that decreased level of 15-LOX and raised level of COX-2 and 12-LOX could indicate a negative prognosis for a cancer patient. There is a growing evidence that i) many oxygenases can be present concurrently in the same tissue, ii) the metabolites from oxygenases pathways and iii) the inhibitors of oxygenases make a tremendous impact on carcinogenesis, metastasis and apoptosis $(1,12,20)$. Thus coexistence of oxygenases is not aberrant but typical. While the high 12-LOX and low 15-LOX levels might be typical for the advanced cancer, the decreased 12-LOX and increased 15-LOX might be an equally poor prognosis at the onset of the disease. Those levels should be compared against normal values established for cancer-free individuals, albeit this could also be highly controversial. Oxygenases are active in many diseases (inflammatory, cardiovascular and more) and we do not know how it might impact the level of enzymes in a studied tissue, thus the normal tissue group (NK) should only contain samples from carefully screened healthy individuals.

Our pilot study is the first to examine the presence of three lipoxygenases simultaneously in the same renal tissue. The samples were segregated into groups containing the same type of cancer either renal clear cell carcinoma or renal papillary carcinoma. We used highly specific antibodies for the detection of the three lipoxygenases (5-, 12S- and 15-LOX-1), confirming the lack of their cross-reactivity. In our study, none of the lipoxygenases appeared suitable to be used individually as a biomarker. However, measuring level of their expression and looking out for the trend in which there is a decline in 12-LOX (and/or 5-LOX) accompanied by an increase in 15-LOX-1 could be indicative of the onset of cancer, but this should be confirmed in a much larger cohort.

There are no biomarkers to individualize therapy in renal cell carcinoma. Unlike other malignancies (for example lung cancer) a tumor specific biomarker has not been identified to predict outcome of therapy in the advanced carcinoma and poor-risk patients. Novel therapeutics, very often tyrosine kinases inhibitors (TKI therapy, for example drug sunitinib) are targeting the vascular endothelial growth factor (VEGF) 
and endothelial growth factor receptor (EGFR), although proteins detected in blood, including VEGF, are not suitable as predictors of RCC treatment. Hypertension is observed as a side effect of VEGF-targeted therapy and could be an indicator of efficacy for several tyrosine kinase inhibitors (including sunitinib) in RCC treatment (21). It has been found that patients who develop hypertension (systolic blood pressure $\geq 140$, diastolic >90) with sunitinib treatment have improved clinical outcomes without clinically significant increases in hypertension-associated adverse events, supporting its viability as an efficacy biomarker $(21,22)$. There is a strong interdependence between 12S-LOX, tyrosine kinases and VEGF pathways. It is known that patients with hypertension have increased level of 12S-HETE secretion in urine (22). While monitoring blood pressure is easy it does not give any insight in the molecular activity. We hypothesize that probing the platelet 12-LOX activity and monitoring its natural metabolite in urine might provide an opportunity to aid in directing the patients treatment, if the fluctuation in its level could be correlated to the action taken and responses in patient's clinical status.

\section{Acknowledgements}

This study was entirely supported by the grant from the Frederick M. Douglass Foundation. The authors wish to thank Ms. M.D. Xi and Dr S. Khuder for their time and helpful remarks on this study.

\section{References}

1. Chow WH, Dong L and Devesa S: Epidemiology and risk factors for kidney cancer. Nat Rev Urol 7: 245-257, 2010.

2. American Cancer Society: Cancer Facts and Figures 2011 American Cancer Society, Atlanta, 2011.

3. Heng DY, Xie W, Bjarnason GA, et al: Progression-free survival as a predictor of overall survival in metastatic renal cell carcinoma treated with contemporary targeted therapy. Cancer 117: 2637-2642, 2011.

4. Berglund L, Björling E, Oksvold P, et al: A genecentric Human Protein Atlas for expression profiles based on antibodies. Mol Cell Proteomics 7: 2019-2027, 2008.

5. Rhodes DR, Kalyana-Sundaram S, Mahavisno V, et al: Oncomine 3.0: genes, pathways, and networks in a collection of 18,000 cancer gene expression profiles. Neoplasia 9: 166-180, 2007.
6. Pontén F, Jirström K and Uhlen M: The Human Protein Atlas - a tool for pathology. J Pathol 216: 387-393, 2008.

7. Haeggström JZ and Funk CD: Lipoxygenase and leukotriene pathways: biochemistry, biology, and roles in disease. Chem Rev 111: 5866-5898, 2011.

8. Skrzypczak-Jankun E, Chorostowska-Wynimko J, Selman SH and Jankun J: Lipoxygenases - a challenging problem in enzyme inhibition and drug development. Curr Enzym Inhib 3: 119-132, 2007.

9. Kulkarni AP: Lipoxygenase - a versatile biocatalyst for biotransformation of endobiotics and xenobiotics. Cell Mol Life Sci 58: 1805-1825, 2001.

10. Fleming ID, Cooper JS, Henson DE, et al (eds.): AJCC Cancer Staging Manual. Lippincott-Raven, Philadelphia, 1997.

11. Sibony $\mathrm{M}$ and Vieillefond A: Non-clear cell renal cell carcinoma. 2008 update in renal tumor pathology. Ann Pathol 28: 381-401, 2008 (In French).

12. Wang D and Dubois RN: Eicosanoids and cancer. Nat Rev Cancer 10: 181-193, 2010.

13. Faronato M, Muzzonigro G, Milanese G, et al: Increased expression of 5-lipoxygenase is common in clear cell renal cell carcinoma. Histol Histopathol 22: 1109-1118, 2007.

14. Matsuyama M,Yoshimura R, Mitsuhashi M, et al: 5-Lipoxygenase inhibitors attenuate growth of human renal cell carcinoma and induce apoptosis through arachidonic acid pathway. Oncol Rep 14: 73-79, 2005.

15. Yoshimura R, Inoue K, Kawahito Y, et al: Expression of 12-lipoxygenase in human renal cell carcinoma and growth prevention by its inhibitor. Int J Mol Med 13: 41-46, 2004.

16. Philips BJ, Dhir R, Hutzley J, Sen M and Kelavkar UP: Polyunsaturated fatty acid metabolizing 15-Lipoxygenase-1 (15-LO-1) expression in normal and tumorigenic human bladder tissues. Appl Immunohistochem Mol Morphol 16: 159-164, 2008.

17. Jack GS, Brash AR, Olson SJ, et al: Reduced 15-lipoxygenase-2 immunostaining in prostate adenocarcinoma: correlation with grade and expression in high-grade prostatic intraepithelial neoplasia. Hum Pathol 31: 1146-1154, 2000.

18. Jiang WG, Douglas-Jones A and Mansel RE: Levels of expression of lipoxygenases and cyclooxygenase- 2 in human breast cancer. Prostaglandins Leukot Essent Fatty Acids 69: 275-281, 2003.

19. Yoshimura R, Matsuyama M, Tsuchida K, Kawahito Y, Sano H and Nakatani T: Expression of lipoxygenase in human bladder carcinoma and growth inhibition by its inhibitors. J Urol 170: 1994-1999, 2003

20. Brown HA and Marnett LJ: Introduction to lipid biochemistry, metabolism, and signaling. Chem Rev 111: 5817-6512, 2011.

21. Rini BI, Cohen DP, Lu DR, et al: Hypertension as a biomarker of efficacy in patients with metastatic renal cell carcinoma treated with sunitinib. J Natl Cancer Inst 103: 763-773, 2011.

22. González-Núñez D, Claria J, Rivera F and Poch E: Increased levels of 12(S)-HETE in patients with essential hypertension. Hypertension 37: 334-338, 2001. 